45 (1) | 2016

En el río del Maíz (Madre de Dios)

Homenaje a Bernard Lelong

\title{
Desde los lamistas hasta la expedición al río del Maíz
}

Jean-Pierre Chaumeil

\section{CpenEdition}

\section{Journals}

Edición electrónica

URL: http://journals.openedition.org/bifea/7753

DOI: $10.4000 /$ bifea. 7753

ISSN: 2076-5827

\section{Editor}

Institut Français d'Études Andines

\section{Edición impresa}

Fecha de publicación: 1 abril 2016

Paginación: 9-15

ISSN: 0303-7495

\section{Referencia electrónica}

Jean-Pierre Chaumeil, « Desde los lamistas hasta la expedición al río del Maíz », Bulletin de l'Institut français d'études andines [En línea], 45 (1) | 2016, Publicado el 08 abril 2016, consultado el 05 noviembre 2020. URL : http://journals.openedition.org/bifea/7753 ; DOI : https://doi.org/10.4000/ bifea.7753

\section{(c) $(1) \&$}

Les contenus du Bulletin de l'Institut français d'études andines sont mis à disposition selon les termes de la licence Creative Commons Attribution - Pas d'Utilisation Commerciale - Pas de Modification 4.0 International. 


\title{
Desde los lamistas hasta la expedición al río del Maíz
}

\author{
Jean-Pierre Chaumeil*
}

Bernard Lelong dedicó buena parte de su vida a América Latina, caminando por los senderos y trochas. En Argentina, se familiarizó con el americanismo y con la escritura. En búsqueda siempre de nuevos horizontes, emprendió muchos viajes en las tierras altas y bajas de Suramérica. Pero es a los lamistas —un pueblo de lengua quechua de 15000 personas - que consagró la mayor parte de sus años de investigación (algo como veinte años) y con los cuales realizó su trabajo antropológico de mayor envergadura (aunque por lástima quedándose inédito).

\section{LOS LAMISTAS}

En un capítulo de su libro redactado con Jean-Luc Lancrey-Javal, Cordillère magique (1955), Bernard Lelong relata su primer viaje entre los lamistas. Esta misión de estudio que ha reunido ambos viajeros a inicios de 1953 recibió las recomendaciones del profesor Paul Rivet, uno de los fundadores del Museo del Hombre de París. En efecto, el profesor Rivet había aconsejado a los dos jóvenes estudiar dos sociedades indígenas de la Amazonía peruana sobre las cuales se sabía muy poco: los cholones y los jibitos. El Centro Nacional de Investigación Científica de Francia (CNRS) apoyó dicho proyecto y la pequeña expedición se puso en marcha el 10 de mayo de 1953.

Dejando de lado las recomendaciones de Rivet de estudiar a los cholones y jibitos, se dedicaron al estudio de los lamistas del río Mayo. En su libro de 1955, Bernard

* Centro EREA del Laboratoire d'Ethnologie et de Sociologie Comparative-LESC, Université Paris Ouest Nanterre La Défense-CNRS. E-mail: jpc@vjf.cnrs.fr 
Lelong nos ofrece una primera descripción de este pueblo. Fascinado por la amplitud de sus conocimientos naturalistas, Bernard Lelong se interesó en particular por su concepción de las enfermedades, por su chamanismo y por el uso que hacían de plantas medicinales, y por los cuales este pueblo gozaba de mucho renombre en todo el departamento de San Martín. Logró constituir un impresionante fichero (no publicado) de más de 350 plantas medicinales que, por su precisión y riqueza, representa sin duda uno de los puntos fuertes de sus trabajos de investigación. Sin embargo, a pesar de todo, reconocía que su fichero era poca cosa frente a los miles de plantas que conocen y utilizan los chamanes lamistas. Este pueblo tuvo notoria influencia sobre la vida y la trayectoria de Bernard Lelong, tanto en el plano personal como profesional, de tal forma que le era difícil esconder, según su propia expresión, un cierto «etnocentrismo lamista». De igual modo, solía autocalificarse, quizás por exceso de modestia o por rechazo a un cierto conformismo académico, de «agente de comunicación interchamánica», en vez de presentarse sencillamente como antropólogo. Sea lo que fuere, adquirió una real expertise y un conocimiento fino en este campo de investigación relativo a los lamistas.

A principio de 1971, le llegó la noticia por la prensa de la desaparición de una expedición francesa en la Amazonía. En París se buscaban voluntarios para emprender un viaje de rescate sobre la suerte de tan desgraciada expedición. Bernard Lelong aceptó el reto de organizarla, sabiendo por experiencia las dificultades (financieras y otras) que surgen en la organización en poco tiempo (unos meses) de una empresa de tal magnitud.

\section{LA EXPEDICIÓN PERDIDA}

Verano 1970 - Tres viajeros desaparecen en el Alto Madre de Dios. Según se cuenta, buscaban una ciudad perdida...

Bernard Lelong y sus compañeros intentan encontrar sus huellas y surcan el Shinkibeni, el río del Maíz....

Cuzco, agosto de 1970

Tres compañeros de larga ruta se juntaron en esta ciudad para formar una expedición que tenía como objetivo explorar el río Shinkibeni (o «río del Maíz»). iDejaban sin embargo entender a los que mostraban más curiosidad que iban de repente a buscar el famoso reino del Paititi! Recordemos que la búsqueda de ciudades perdidas en el corazón de la selva amazónica ha sido y sigue siendo viva - casi sin interrupción desde los primeros tiempos de la colonización europea hasta hoy en día - en estas regiones lejanas del departamento de Madre de Dios. La expedición integraba a Serge Debru y Gérard Puel (dos franceses de 29 y 31 años respectivamente) y a Bob Nichols, un periodista norteamericano de 37 años, corresponsal en Lima de la revista Peruvian Times. La expedición salió de la misión dominicana de Shintuya (Alto Madre de Dios) el 30 de agosto de 1970 
con dos guías amarakaeris (perteneciendo al conjunto harakmbut) que se supone conocían la región y entendían algo del idioma matsiguenga (lengua arawak subandina). El 20 de setiembre de 1970, isorpresa!: los dos guías regresaron a la misión de Shintuya sin los tres viajeros, llevando por todo testimonio un casete grabado donde los expedicionarios narraban las dificultades del viaje y la falta de alimento. Este documento fue publicado en el Peruvian Times de febrero de 1971. Desde este momento, silencio: no se tuvo más noticias de los viajeros.

Varias expediciones de rescate fueron organizadas, la primera en mayo de 1971 coordinada por la misión de Shintuya y la segunda financiada por el Ministerio de Asuntos Exteriores de Francia, bajo recomendaciones del Primer Ministro de la época, y encabezada por un cierto Nicolas Asheshov en coordinación con un colono de apellido Berg, especialista en la lucha antiguerrilla. Se publicaron varios reportes de Asheshov en el Peruvian Times (Asheshov, 1971a-f). Sin embargo, ninguna de las dos tuvo éxito.

Una expedición posterior, sin mayor éxito tampoco, fue organizada en 1973 por el explorador japonés Yoshitaro Sekino, quién publicó varias fotografías en el Peruvian Times de los supuestos asesinos (atribuido a los matsiguengas) de la expedición Debru, pero sin mucha prueba.

Sea lo que fuere, en setiembre de 1971, salió de la misión de Shintuya la expedición encabezada por Bernard Lelong.

\section{EN EL RÍO DEL MAÍZ}

El equipo se componía de tres personas: Bernard Lelong, JeanClaude Tann, un amigo personal de Serge Debru, y Pierre Fattori, camarógrafo encargado de realizar una película con el fin de rentabilizar los gastos de la expedición.

El relato tal como aparece en el libro (Lelong, 1974) contempla dos periodos. Una primera parte concierne el viaje hasta los petroglifos de la roca Pusharo que, se supone, marca el límite del territorio matsiguenga. Es conocido que estas inscripciones sobre la roca fueron descubiertas por el padre dominico Cenitagoya hacia 1921. La segunda parte relata la difícil surcada del río Shinkibeni, afluente del Madre de

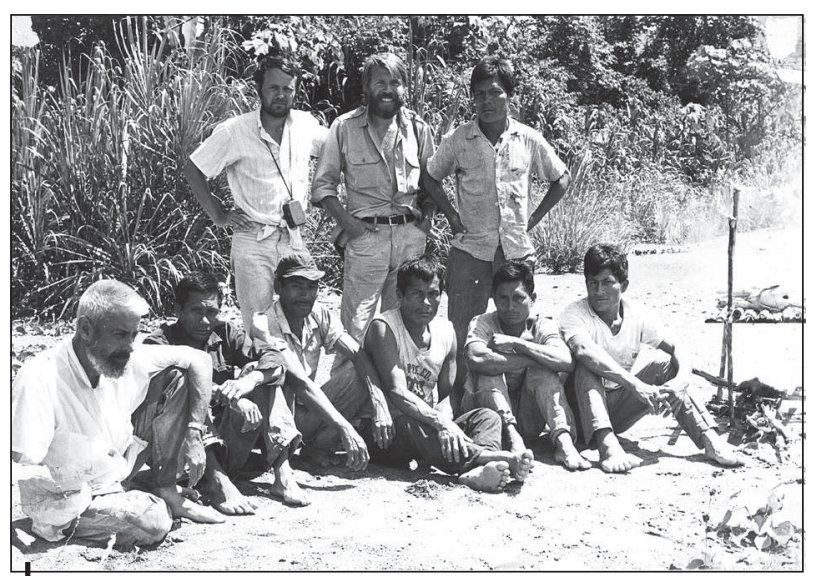

Figura 1 - Los miembros de la expedición

De pie: Jean-Claude Tann, Pierre Fattori y un guía amarakaeri; sentado: Bernard Lelong al lado de los cinco otros guías amarakaeris

(C) J.-C. Tann, 1971

Dios. 


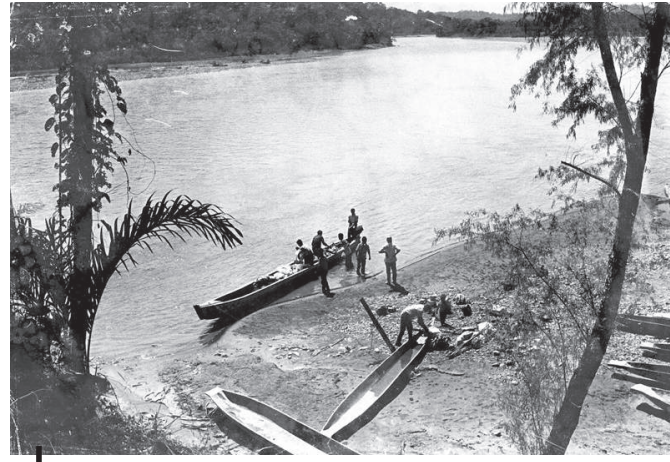

Figura 2 - Embarcadero para alistar las canoas de surcada

(c) J.-C. Tann, 1971

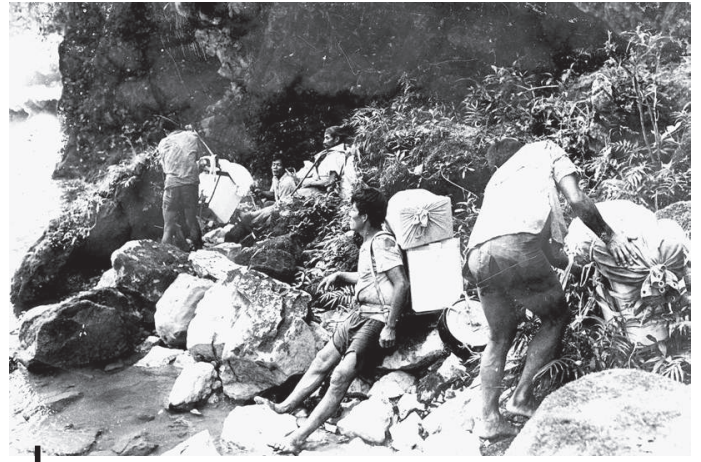

Figura 3 - Cargadores amarakaeris en el camino hacia el Alto Shinkibeni

(C) J.-C. Tann, 1971

El tono narrativo de Lelong, en su descripción del viaje, se vuelve a menudo lírico y entrecortado por frecuentes digresiones filosóficas o seudofilosóficas con referencias a autores tan distintos como Deleuze y Guattari, el Cheik Hamidou Kane, Laurence de Arabia, André Malraux, Franz Fanon o Robert Jaulin y su Paz blanca, así como a sus amigos cercanos, Stefano Varese (del cual tradujo al francés varios textos en la revista Les Temps Modernes), Jacques Meunier o Simone Waisbard, para citar solo a algunos. Un tema que surge de manera repetitiva (casi obsesiva) a lo largo de las páginas, y que contrasta con sus reflexiones de corte digamos más especulativa, es su rechazo rotundo de la obra misionera contra la libertad de los indígenas, con un tono incluso molesto y amargo al momento de referirse a la misión de Shintuya. Al mismo tiempo dedicó largos pasajes a temas que quizás podríamos calificar de triviales como cuando se lanza en la descripción de su famoso blue-jean todo parcheado de piezas cosidas, cada una recordando «un evento extraordinario de la vida y experiencias con la ayahuasca» (Lelong, 1974: 107).

Sea lo que fuere, la expedición llega a la roca Pusharo el 14 de septiembre.

A partir de este punto la surcada se vuelve más difícil y peligrosa por la fuerte corriente debido a las intensas lluvias de los últimos días y el paso de los pongos.

El 17 de septiembre llegan por fin al último campamento y punto de encuentro de la expedición Debru con un grupo de matsiguengas (kogapakori): localizar a este grupo se vuelve para los expedicionarios la última oportunidad para tener noticias sobre la suerte de Debru y de sus compañeros.

El contacto se establece poco después y el pequeño grupo de matsiguengas pone

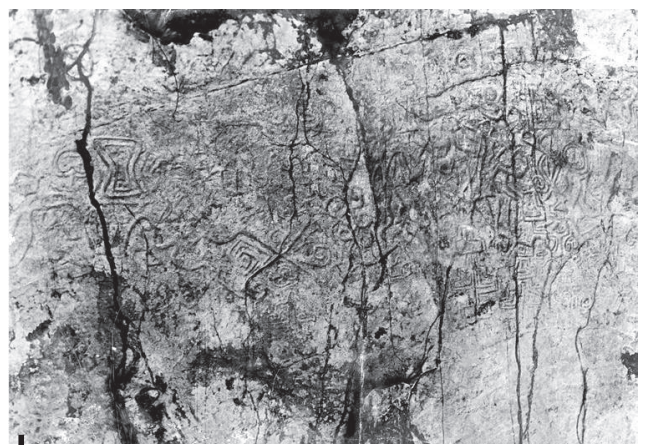

Figura 4 - Petroglifos sobre la roca Pusharo, marcando el límite del territorio matsiguenga

(C) J.-C. Tann, 1971 
a disposición de Lelong y de sus compañeros una casa abandonada después de la muerte de una mujer (sic).

La conversación se establece via Mario (el intérprete amarakaeri). Según la versión que recogieron de los matsiguengas, los tres viajeros habrían muerto de hambre en las cabeceras del Shinkibeni y sus cuerpos habrían sido llevados por la corriente. No quedarían huellas de ellos ni tampoco noticias de la existencia de hipotéticas ruinas en la región que hubiera podido motivar una estadía más larga en la zona. Frente a la certeza de la

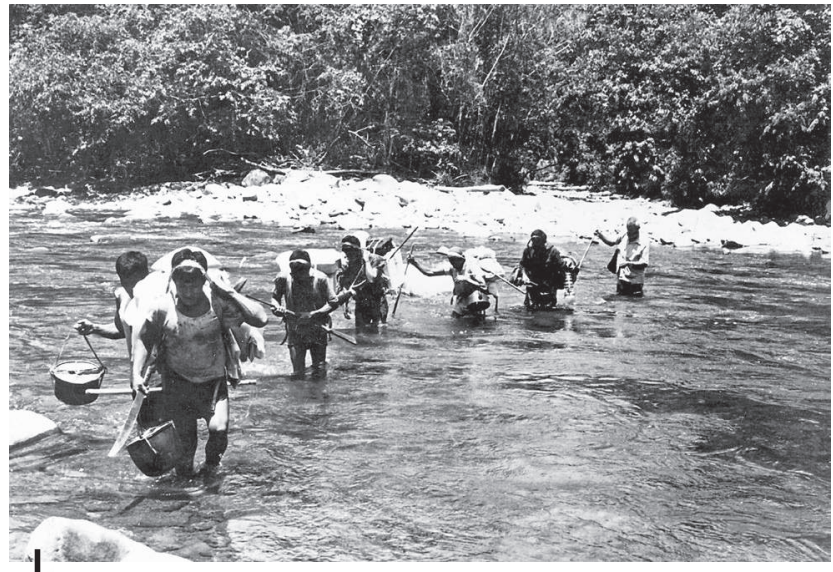

Figura 5 - Cruzando el río del Maíz, camino hacia el Alto Shinkibeni

(C) J.-C. Tann, 1971 muerte de los tres viajeros en el transcurso del mes de noviembre de 1970, no quedo más a los expedicionarios que volver a la misión de Shintuya para informar sobre su viaje.

iLo sorprendente es que la misión dominicana no dudo en confirmar, sin mucha prueba (EI Comercio del 18 de enero de 1972) la tesis de la muerte de los viajeros a manos de los matsiguengas!, tesis sostenida a su vez por el explorador japonés Yoshiharo Sekino en el Peruvian Times de noviembre de 1973 (Sekino, 1973a; b)... pero sin mayor prueba tampoco. Probablemente nunca se sabrá lo que realmente pasó en este mes de noviembre de 1970 en el Alto Shinkibeni.

\section{POST-SCRIPTUM}

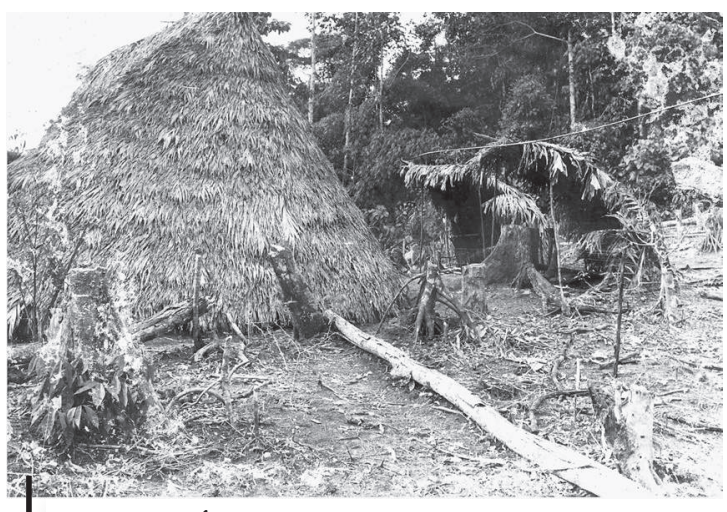

Figura 6 - Última casa matsiguenga encontrada en el Alto Shinkibeni

(c) J.-C. Tann, 1971
Al retorno de su expedición, Bernard Lelong dedicó gran parte de su tiempo al montaje de la película sobre el viaje y a la redacción del libro La rivière du maíz (publicado en 1974 sin fotografías) para dar cuenta de los sucesos en el Shinkibeni. No cabe duda también que Bernard proyectaba la redacción de su gran obra sobre los lamistas y sus conocimientos medicinales, incluyendo al famoso fichero de plantas que él elaboró a lo largo de muchos años. Pero el destino y las vicisitudes de la vida no le permitieron realizar su proyecto. Sin embargo, su memoria se perpetúa a través del legado 


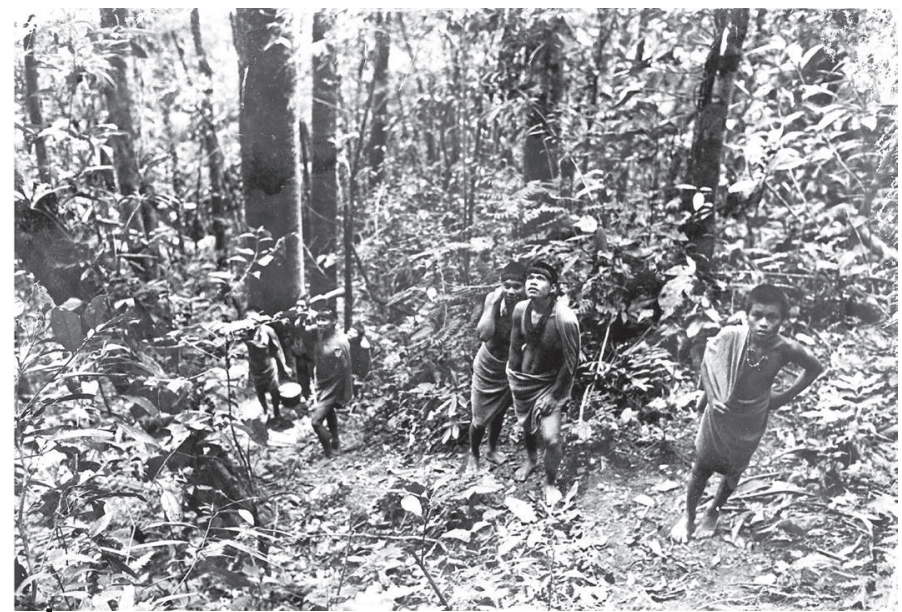

Figura 7 - En la selva, siguiendo la trocha con los matsiguengas

(C) J.-C. Tann, 1971

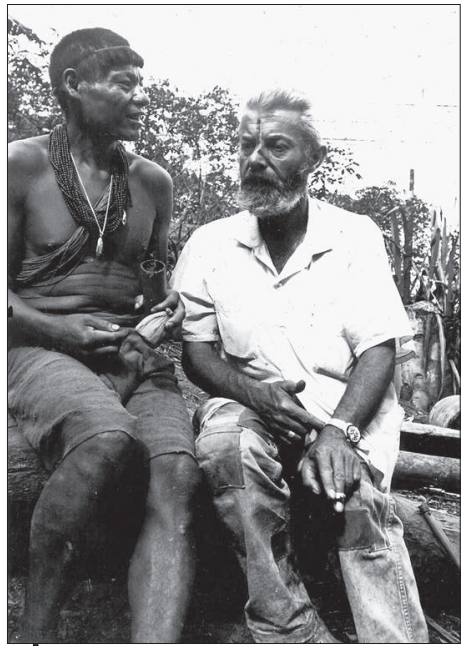

Figura 8 - Bernard Lelong con un indígena matsiguenga utilizando achiote para pintarse la cara

(C) J.-C. Tann, 1971 que hizo al Departamento de Ciencias Humanas del CNRS, hoy Instituto de Ciencias Humanas y Sociales. Esta donación estaba destinada a financiar becas con fines de realizar investigaciones antropológicas sobre las culturas de la Amazonía peruana. Iniciadas en 1984, estas becas permitieron a muchos estudiantes y antropólogos culminar sus tesis o llevar a cabo sus proyectos de investigación. El presente volumen es un buen ejemplo de la importancia y beneficio de este legado.

Hoy, el archivo etnográfico de Bernard Lelong se encuentra depositado en el Centro EREA del LESC (Laboratorio de Etnología y Sociología Comparativa de la Universidad de Paris Ouest Nanterre La Défense y del CNRS). Por primera vez, se reproduce arriba algunas fotografías tomadas durante la expedición al río del Maíz que dan ideas de las condiciones del viaje, haciéndonos penetrar un instante en las húmedas atmosferas y soledades del Alto Madre de Dios. 


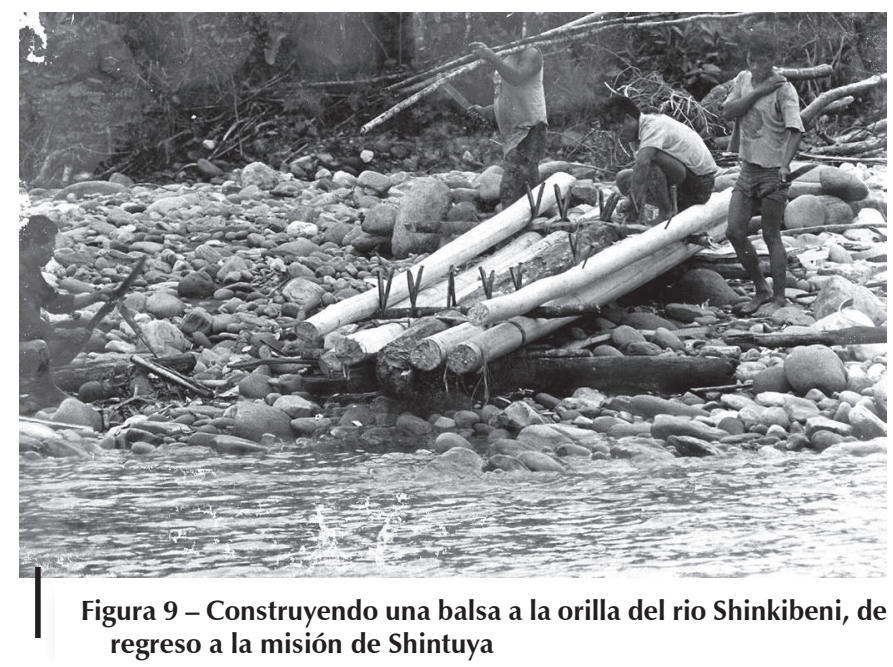

(c) J.-C. Tann, 1971

\section{Referencias citadas}

ASHESHOV, N., 1971a - The lost Pantiacolla Expedition. Search party sets out from Shintuya. A special Report by Nicolas Asheshov. Peruvian Times, 14 de mayo: 8-9.

ASHESHOV, N., 1971b - Search party penetrating the unknown Pantiacolla. Peruvian Times, 21 de mayo: 9-11.

ASHESHOV, N., 1971c - Search for lost explorers covered from air. Peruvian Times, 4 de junio: 3.

ASHESHOV, N., 1971d - Air Search for missing explorers draws blank: ground party finds equipment cache. Peruvian Times, 11 de junio: 3.

ASHESHOV, N., 1971e - Search party account. Ridge camp Discovery. Peruvian Times, 25 de junio: 4-5.

ASHESHOV, N., 1971f - Second Search for lost Pantiacolla explorers finds nothing. Peruvian Times, 22 de octubre.

LELONG, B., 1974 - La rivière du maïs. A la recherche d'une expédition perdue en I'Amazonie, 225 pp.; París: Edition J-C.Lattès.

LELONG, B. \& LANCREY-JAVAL, J.-L., 1955 - Cordillère magique, 229 pp.; París: AmiotDumont (bibliothèque des voyages).

SEKINO, Y., 1973a - The Wilderness of the Pantiacolla Jungles. Peruvian Times, 23 de noviembre: 15-18.

SEKINO, Y., 1973b - The Machiguengas of the Pantiacolla; "Why we killed the three viracochas". Peruvian Times, 30 de noviembre: 11-16. 